\title{
FIBROUS TISSUE RING: AN UNCOMMON CAUSE OF SEVERE PROSTHETIC VALVE STENOSIS
}

\author{
Paolo Masiello, MD, Vincenzo Cassano, MD, and Giuseppe Di Benedetto, MD, Salerno, Italy
}

We describe here the case of a patient in whom severe mitral stenosis and periprosthetic leak developed 5 years after mitral valve replacement with a Medtronic Hall prosthesis (Medtronic, Inc., Minneapolis, Minn.). Mitral valve stenosis was attributed to the formation of concentric dense fibrous tissue around the atrial side of the anulus.

A 45-year-old woman underwent open mitral commissurotomy in 1979 for rheumatic mitral stenosis. Ten years later she underwent mitral valve replacement with a Medtronic Hall $27 \mathrm{~mm}$ prosthesis. In 1993 she had a fever of unknown origin. Subsequently her clinical status progressively worsened, with the onset of exertional dyspnea and fatigue. In March 1994 she was referred to us. On admission severe peripheral edema and jugular vein distention were present. The liver was palpable $6 \mathrm{~cm}$ below the costal border. A $2 / 6$ to $3 / 6$ soft holosystolic murmur was audible on the apex radiating to the axilla. Blood pressure was $130 / 80 \mathrm{~mm} \mathrm{Hg}$. The electrocardiogram showed sinus rhythm with a heart rate of 96 beats $/ \mathrm{min}$ and signs of moderate right ventricular hypertrophy. The chest $\mathrm{x}$-ray film revealed a slightly enlarged cardiac shadow and pulmonary congestion. A transthoracic two-dimensional and Doppler echocardiogram showed left atrial dilatation, normal opening of the prosthesis, reduction of diastolic flow through the prosthesis, and a mild periprosthetic leak. High pulmonary artery pressures were calculated and then confirmed by preoperative insertion of a thermodilution catheter $(90 / 45 \mathrm{~mm} \mathrm{Hg}$, mean $60 \mathrm{~mm} \mathrm{Hg})$. A transesophageal echocardiogram revealed a periprosthetic leak and confirmed the transthoracic data. Mild aortic stenosis was present.

The patient was operated on 2 days later and the mitral prosthesis was replaced through a vertical transseptal approach with a $27 \mathrm{~mm}$ CarboMedics prosthesis (CarboMedics, Inc., Austin, Tex.). The aortic valve was explored and subsequently a commissurotomy was made. The postoperative course was uneventful and the patient was discharged to her home on the ninth postoperative day. Postoperative transthoracic two-dimensional and Doppler echocardiography showed a dramatic drop in pulmonary artery pressure and a well-functioning prosthesis.

Examination of the explanted valve revealed a ring of fibrosclerotic tissue, containing many inflammatory cells, surrounding the anulus on the atrial side. The movement of the disc was unimpeded but the fibrous tissue strongly reduced the valvular area, simulating mitral stenosis (Fig. $1, A$ and $B$ ).

From the Department of Cardiac Surgery, Hospital "S. Leonardo," Via S. Leonardo 1, 84100, Salerno, Italy.

J THORAC CARdiovasC SURG 1995;109:1253

Copyright (C) 1995 by Mosby-Year Book, Inc.

$0022-5223 / 95 \$ 3.00+0 \quad \mathbf{1 2 / 8 / 5 7 9 9 3}$
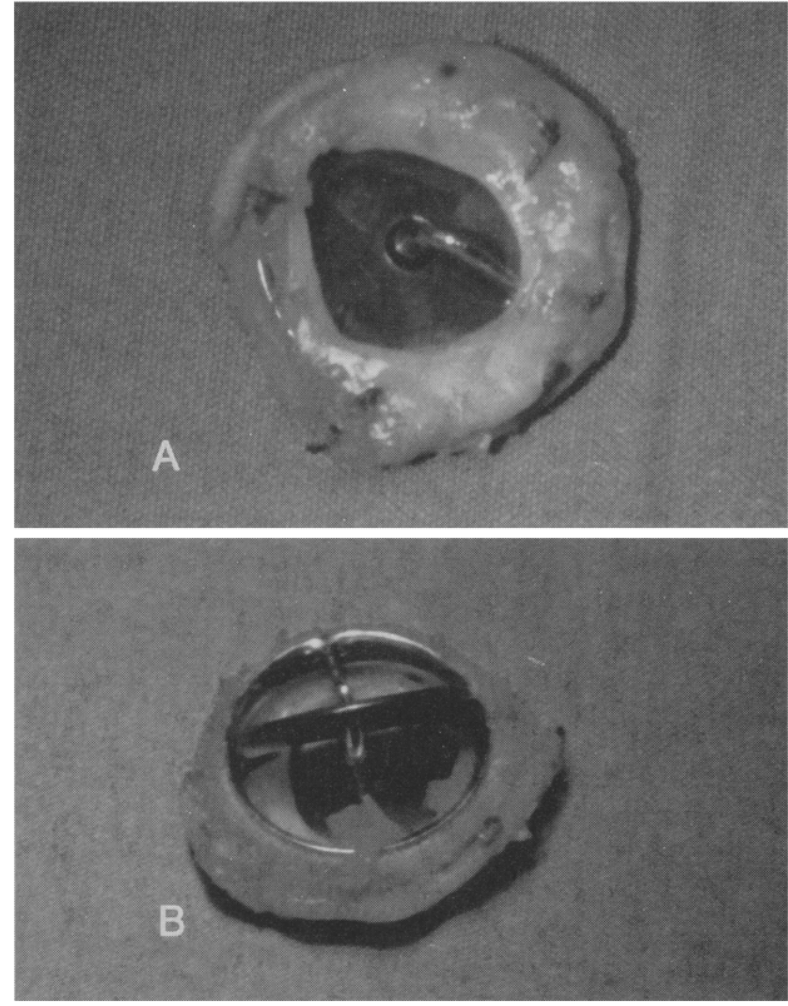

Fig. 1. Explanted mitral prosthesis. A, Atrial view. B, Ventricular view.

Prosthetic valve endocarditis strongly predisposes the patient to periprosthetic leakage; when it occurs, valve replacement is indicated. ${ }^{1,2}$ In our case infective endocarditis probably was the cause of the leakage and of the formation of the fibrous ring. Reoperation was done because of the leakage, but the regurgitation was mild and could not explain the severity of the symptoms and of the pulmonary hypertension. Reduction of the mitral area, however, was severe.

Formation of inflammatory tissue after prosthetic valve endocarditis greatly reducing the valvular area is uncommon; when it happens, reoperation is indicated.

\section{REFERENCES}

1. Kirklin JW, Barratt-Boyes BG. Cardiac surgery. 2nd ed. New York: Churchill Livingstone, 1993.

2. Blackstone EH, Kirklin JW. Death and other timerelated events after valve replacement. Circulation $1985 ; 72: 753-67$. 